\title{
X-ray Laser Developments at PHELIX
}

\author{
B. Ecker ${ }^{1,2}$, B. Aurand ${ }^{2,3,4}$, D. C. Hochhaus ${ }^{3,7}$, P. Neumayer ${ }^{3}$, B. Zielbauer ${ }^{1,4}$, K. Cassou $^{5}$, S. Dabous-

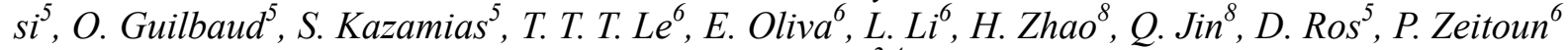 \\ and T. Kuehl,
}

${ }^{1}$ Helmholtz Institute Jena , ${ }^{2}$ Johannes-Gutenberg Universität Mainz, ${ }^{3}$ ExtreMe Matter Institute EMMI, ${ }^{4}$ GSI Helmholtzzentrum fuer Schwerionenforschung, ${ }^{5}$ LASERIX-CLUPS / Université Paris-Sud 11 ${ }^{6}$ Laboratoire d'Optique Appliquée, ${ }^{7}$ Goethe-Universität Frankfurt, ${ }^{8}$ Institute of Modern Physics, CAS

\section{Introduction}

We report on results of a double-stage molybdenum x-ray laser experiment. The two targets were pumped using the double-pulse grazing incidence pumping technique, which includes travelling wave excitation for both the seed- and the amplifier-target..

The main motivation for X-ray laser (XRL) research at GSI is to perform spectroscopy experiments on highlycharged heavy-ions stored in the experimental storage ring (ESR) of the GSI accelerator facility[1]. The first experiment of this kind will aim at measuring the $2 \mathrm{~s} 1 / 2$ $2 \mathrm{p} 1 / 2$ transition in Li-like ions. For ions of an atomic number between $50(\mathrm{Sn})$ and $92(\mathrm{Ur})$, this transition energy lies between $100 \mathrm{eV}$ and $300 \mathrm{eV}$ [1], which corresponds to wavelengths between $12 \mathrm{~nm}$ and $4 \mathrm{~nm}$. Setting up the experiment in a way, where the XRL is counter propagating to the ion bunch, one can exploit the relativistic Doppler effect. The use of laser-pumped plasma XRL's, with typical photon energy up to $100 \mathrm{eV}[2,3,3]$ can address the whole range of lithium-like ions for the lowest lying transitions The perspective for FAIR, given by the even higher ion velocities at HESR, opens a completely new range of experiments.

\section{Experiment}

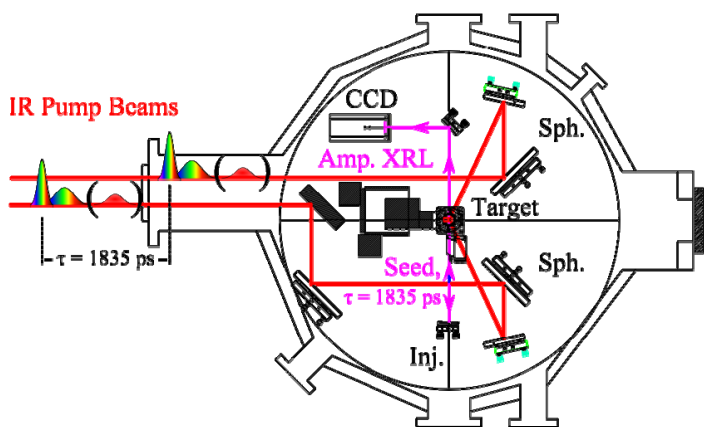

Fig. 1. Sketch of the experimental setup. A more detailed description is given in the text.

A Mach-Zehnder like interferometer, which was implemented in the short-pulse frontend of the PHELIX laser, was used to create the chirped double-pulse structure required for the DGRIP scheme [2]. After compression, the pulse duration of the two pulses was 200 ps (prepulse) and 2 ps (main pulse). Using the PHELIX pre- amplifier section, the total pump energy on the target amounted to $600 \mathrm{~mJ}$, equally distributed between two individual pumping beams. Inside the target chamber, the two beams were focused in opposite direction onto the Mo slab target by two spherical mirrors, as illustrated in Fig. 1. The line foci were vertically separated by $\sim 3 \mathrm{~mm}$. The output of the lower XRL - the seed pulse - was focused into the upper - amplifying- medium by a spherical XUV mirror.

\section{Results}

Seeded x-ray laser operation has been demonstrated, resulting in x-ray laser pulses of up to $240 \mathrm{~nJ}$ and $2 \mathrm{mrad} \times$ $2 \mathrm{mrad}$ divergence. The peak brilliance of the amplified $\mathrm{x}$-ray laser of $4 \times 10^{23}$ photons $/ \mathrm{s} / \mathrm{mm}^{2} / \mathrm{mrad}^{2}$ in $5 \times 10^{-5}$ relative bandwidth was more than two orders of magnitude larger compared to the original seed pulses.Figure 1 shows the typical beam patterns of the $\mathrm{HH}$ observed (filters: $\mathrm{Zr}$ and $\mathrm{Ti}$ ) under (a) only $\mathrm{He}$ gas jet (valve stagnation pressure: 4000 mbar), (b) Ne $400 \mathrm{mbar}$ jet, and (c) both gas jets for $\mathrm{Ne} 400-\mathrm{He} 4000$ mbar.

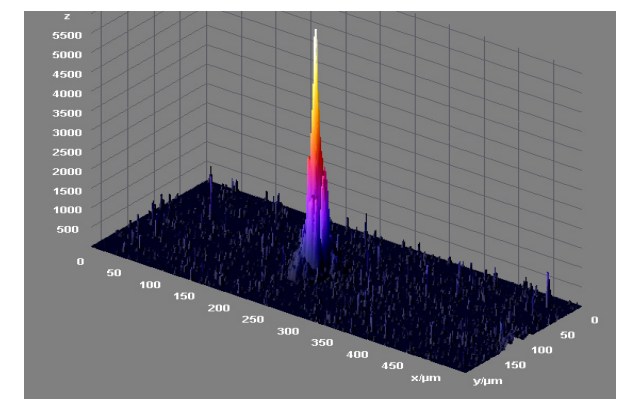

Fig. 2: Beam quality of the seeded XRL. showing $2 \mathrm{mrad} \times 2 \mathrm{mrad}$ divergence.

\section{References}

1. D.Winters et al., GSI Annual report 2012

2. B. Ecker et al., Opt. Express 20(23), 25391 (2012).

3. D. Zimmer et al., Optics Express, 13, 2403, 2008

4.D. Ros et al., NIM A, 653, , 76, 2011 